\title{
Quantum model of the magnetic field of the Hot Expanding Earth
}

\author{
Vladimir Kuznetsov ${ }^{1}$ \\ Institute of Cosmophysical Research and Radio Wave Propagation FEB RAS, \\ 684034, Kamchatka, Paratunka, Mirnaya str., 7, Russia
}

\begin{abstract}
Principally new quantum model of the magnetic field of the Hot Earth [1] is proposed here. The model proceeds from the generation scheme of the Earth magnetic field (EMF) reported at the conference in 2019 [2], where crucial role of quantum entanglement $(\mathrm{QE})$ of the inner G-core matter for EMF generation under the Hall effect was first evidenced. With regard to my impact into geodynamics of the Hot Earth [3] and quantum geophysics [4] the improved version of [2] is presented here. The justification for the model of EMF generation given in the encyclopedia of Geomagnetism [5] due to the daily rotation of the DEL (double electric layer), exciting a weak field and its amplification by the Hall current is first used here.
\end{abstract}

\section{Introduction}

The Earth's gravitational energy $E=3 / 5 G M^{2} / R=2.25 \times 10^{39}$ erg (here: $G$ is the gravitational constant, $M$ - the Earth's mass and $R$ - its radius) [1] necessary to smash the Earth's mass into dusty particles far from each other would come out in the reversal process of the Earth matter compression by selfgravitation yielding its heating. Dividing $E$ by heat capacity of the Earth's substance $\left(c_{p}=0.3 \mathrm{cal} / \mathrm{g} \cdot \mathrm{grad}\right)$ and its mass we see the temperature of the Earth's matter of about $30000 \mathrm{~K}$.

Estimating the initial radius of the Earth $R_{\mathrm{o}}$ one can see that continents can be laid one to another with virtually no gaps on the ball with the radius equal to that of the Earth's outer core of 3.5 thousand kilometers which is the initial radius.

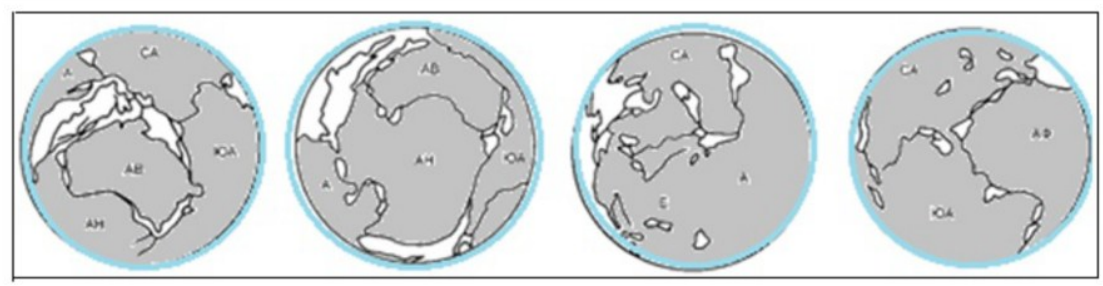

Fig. 1. Continents associating over the globe with a radius of the outer core [1]. Grey - granite Pangaea. Blue - water shell with the temperature of $30^{\circ} \mathrm{C}$. The Earth's age $=500 \mathrm{Myr}$, Cambrian explosion (CE).

\footnotetext{
* Corresponding author:vvkuz38@mail.ru
} 


\section{Hot Earth evolution}

\subsection{Radius evolution}

Time-variation of the Earth's radius from Cambrian explosion (CE) up to nowadays is shown in Fig.2 -a [1].

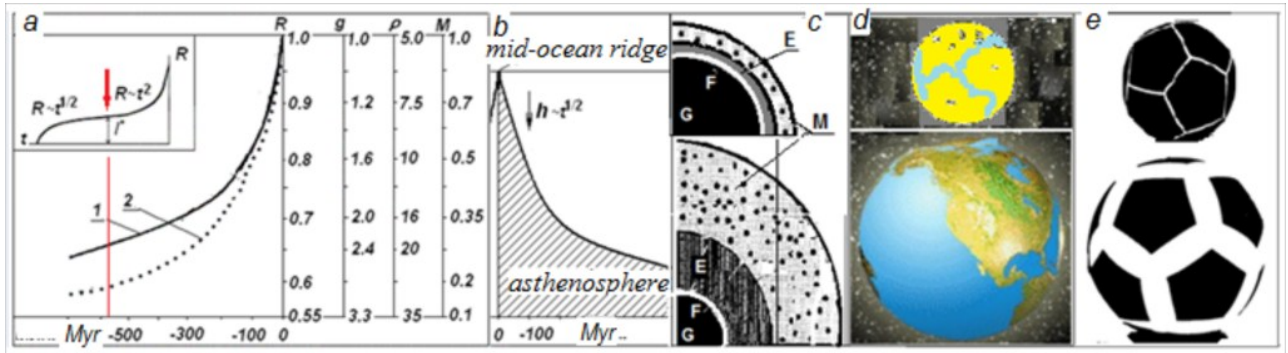

Fig. 2. Time-variation of the Earth's radius $R . a-1,2$ curves show time-variation of $R(1)$ and ocean mass (2) [1], here $g, \varrho, M$ - gravity, ocean density and mass. $b$ - mid-ocean ridge (MOR) cross-section which height $h$ is decreasing with time as a square root. The Earth's radius and the MOR height are interrelated*. $c$ - the Earth's cross section at CE (red arrow at the top) and nowadays (red line at the bottom). $d$-continents at CE, when oceans appeared (at the top) and nowadays (at the bottom). $e-$ the Earth's expanding modeled by Carey [1].

$* R$ and $h$ (asthenosphere thickness) are interrelated. Let's compare the obtained relation $\Delta R$ $\sim t^{2}$ (or $R \sim t^{2}$ ) with the well-known character of $h$ in the spreading zone against the distance from the center of MOR, estimated in years: $h \sim t^{1 / 2}$. These functions are uniquely inverse** and symmetric with respect to $\Delta R \sim t_{R}$, or $h \sim t_{h}$. Indeed, $\Delta R=-h$, and $t_{R}=-t_{h}$. Our estimates for $\left(\Delta R \sim t^{2}\right)$, and $h$ in $\mathrm{km}$ and $t$ in million years, give $h \approx 100 \times t^{1 / 2}$ when $t<500$ million years. Thus, the asthenosphere thickness dependence on the time of evolution (Fig. 2-b) provides (in our model) the rate of the Earth's expanding.

\subsection{Continents}

Following to our model of the hot Earth the initial density of the Earth's matter was about $35 \mathrm{~g} / \mathrm{cm}^{3}$, and the molecular weight $\mu$ was about 10 . It is essential that both core and mantle consist of the same matter (or a mixture of $\mathrm{SiO}_{2}, \mathrm{H}_{2} \mathrm{O}$, and $\mathrm{H}_{2}$ ). The calculated water density at a pressure close to that at the center of the Earth (360 GPA) is shown at the insert, Fig.3-I [6]. The density of $\mathrm{H}_{2} \mathrm{O}$ at $300 \mathrm{GPA}$ is close to $4 \mathrm{~g} / \mathrm{cm}^{3}$. The relationship in logarithmic scale shown in the insert in Fig. 3-I is a straight line. Similar correlations for $\mathrm{H}_{2}$ and $\mathrm{SiO}_{2}$ [6] show that the density of $\mathrm{SiO}_{2}$ at $360 \mathrm{GPA}$ is about the modern density of the inner core $\left(\approx 13 \mathrm{~g} / \mathrm{cm}^{3}\right)$. As evident from our model [1], the initial pressure in the center of the Earth could be 10-15 times higher than the current one providing the initial $\mathrm{SiO}_{2}$ density of about $35 \mathrm{~g} / \mathrm{cm}^{3}$.

The continents are known to consist mostly of granite, while the oceanic crust is made up of basalt. The problem if it is possible to turn basalt into granite and vice versa interested me at the initial stage of developing my model of the Hot Earth. Some geologists agreed that "granitization" of basalts at a high temperatures (about ten thousand degrees), when due to selective evaporation of chemical elements basalt turns into granite of continents, and the remaining primary matter of the Earth into basalt and water are possible (Fig. 3-II). 
The continents (Fig. 1) are torn apart by internal tensile stresses, with the space between them being filled with a mixture (basalt + water) and the coastline (continentocean) which remains unchanged through further expanding of the Earth is formed.

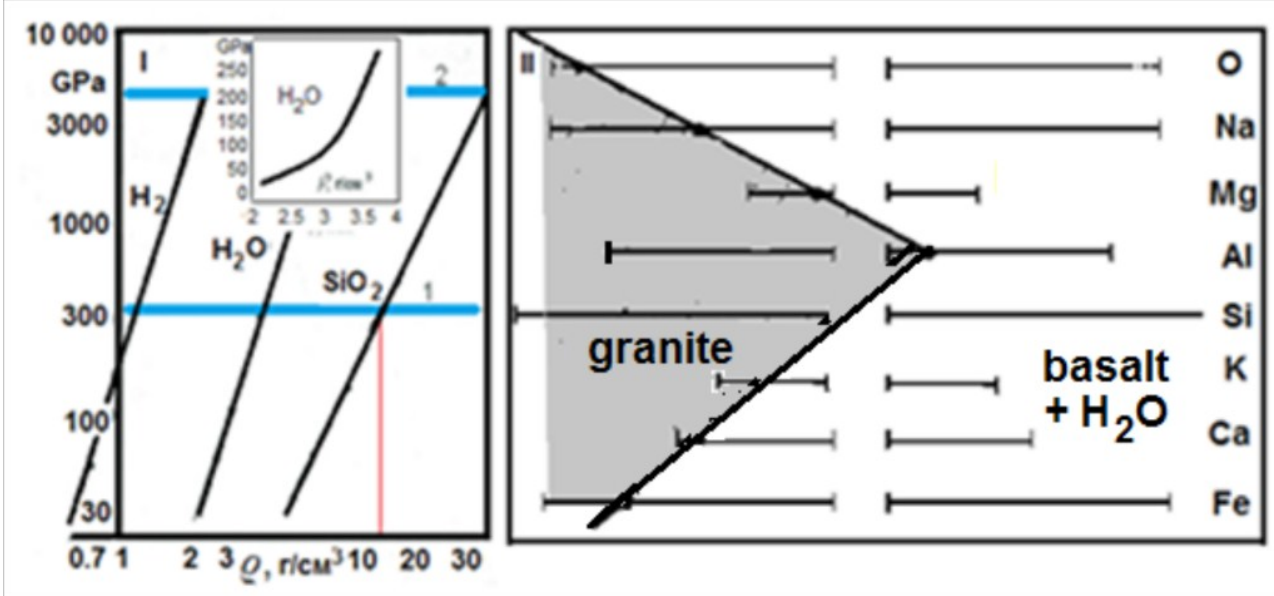

Fig. 3. I - the main components of the Earth (pressure-depending density of $\mathrm{H}_{2}, \mathrm{H}_{2} \mathrm{O}$ and $\mathrm{SiO}_{2}$ ) [6]. Blue line (BL) 1 - pressure in the center of the modern Earth, BL 2 - at the time of its formation. II "granitization" of basalts [1]. As one can see granites contain significantly less water (oxygen) than basalts.

\subsection{Convection in the outer core}

By the Hot Earth model [1], the solid core before its separating into the solid internal and the liquid outer ones consisted of silicon oxide (basalt) with a density of about $35 \mathrm{~g} / \mathrm{cm}^{3}$. Through radiation from its surface the core was cooling from its initial temperature of about $30,000 \mathrm{~K}$ and the molten basalt was turning into molten granite of continents due to the rate differences of evaporation of chemical elements (Fig. 2-C) giving an outer liquid shell and an internal solid core. After the Rayleigh number had been required the convection accelerating the cooling of the core started in the liquid core. Crystallizing at the boundary of the outer core the liquid part of the core was giving the mantle (Fig. 2-b). The inner Gcore remained solid and quantum-entangled. At its boundary, a transition F-layer in which phases divided into liquid and solid ones was formed. In the outer core called the E-core there was always convection. In the F-layer, the sound speed (P-waves) has a break (highlighted in green in Fig. 5-a).

Generally accepted mechanism of the matter convection in the outer E-core is based on kinematic dynamo. A reasonable question, if there is any objective data showing how true this idea of convection is (figures 4-1 and 4-2) arises. The most representative information about the density distribution in the core was obtained from the spectral modes of the Earth's natural vibrations, the so-called splitting functions. Fig. 4-3 shows the splitting function of the ${ }_{11} \mathrm{~S}_{4}$ mode and the possibility of its implementation in the outer core as convection through 12 Rayleigh-Benard convective cells (Fig. 4-4 and 4-5) [7]. Images 4-1 and 4-2 are to be speculative and erroneous! 


\section{The size and location of the source of the EMF generation}

Estimating the size and location of source of the EMF generation we used five independent methods [8]: computer simulation of the geomagnetic field by the set of current rings (current-loop) where the optimal number of rings and the radius of their location were selected; decomposing of the field on the Earth surface into spherical functions and the small parameter which is the ratio of the source size and the distance to it, because the functionsare equivalent; a distance between the virtual magnetic poles (VMP); spatial size of the magnetic anomalies; the location of the source following to the geomagnetic spectrum. The same result that the Earth's magnetic field is generated at the boundary of the inner and outer core in the F - layer was obtained in all estimates [1].

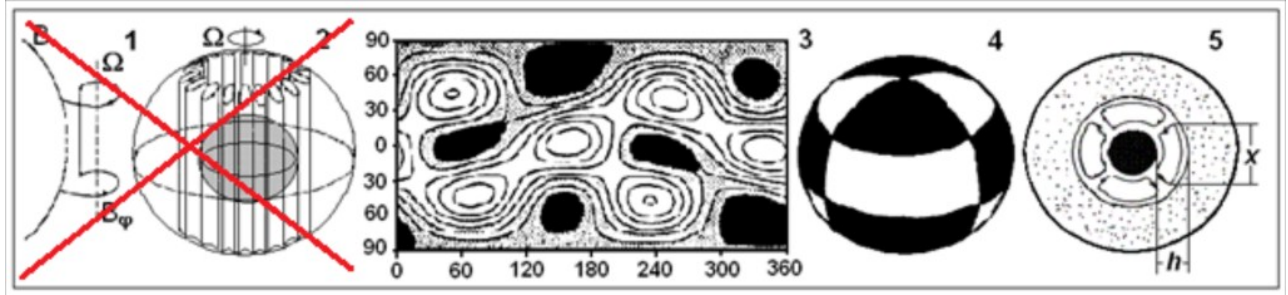

Fig. 4. Convection in the Earth's outer core, two models: 1. - Twisting of the magnetic force line (B) under the differential rotation of the matter $(\Omega$-effect). 2. - $\alpha$ - effect: generation of a meridional field using loops formed by toroidal force lines. 3. - Distribution of the matter density in the outer core, obtained by the spectrum of the core natural vibrations. 4. - Three-dimensional implementation of the matter density distribution in the outer core. 5. - Convection scheme: black circle - the inner core, ring with points - the mantle [7].(Fig. 4-1,2, crossed out with red, occur in almost every textbook or article that discuss the source of the EMF generation. These figures are in obvious contradiction with my proposed model of the EMF generation and I have repeatedly noted that they are not supported by observations, but I have not met with a response. In order to verify this apparent contradiction, more than 20 years ago the Novosibirsk geophysical Observatory conducted a special assessment of the size and location of the source of the EMF generation).

\subsection{Features of the Earth's core according to observations}

Fig. 5-a,- b, - c shows data on the velocity of P- and S- waves propagation, the electrical conductivity and viscosity of the Earth's shells.

By the common model of the cold Earth, the core (iron) conductance is high implying that changes of the geomagnetic field actually registered by magnetologistsshouldn't exit to the surface of the Earth. The problem of the Earth's conductance would be free from contradictions if we assume that the outer core and the mantle consist of the same poorly conducting material and their electrical conductance increase slowly with increasing temperature, and the generation of the geomagnetic field (as follows from the preceding) occurs at the boundary of the inner core, where oincreases sharply. According to our model, the conductance in the F-layer has a tensor character (just like in ionosphere): $\sigma_{R}-$ across the layer and $\sigma_{\varphi}-$ along it. As will be shown later, $\sigma_{R} d e p e n d s$ on polarization and separation of electric charges in the F-layer, and the increased conductance $\sigma_{\varphi}$ of the mobile Ekman layer (geostrophic wind) is apparent from the matter properties at the critical temperature. Under critical $p T$ conditions the matter is known to be "superfluid" and "superconductive". The conductance of the matter in the inner core $\sigma_{\mathrm{G}}$ is the highest. 


\subsection{The viscosity of the matter in the outer core}

As the viscosity of the outer core is effective to transmit rotation to the inner core the author [11] estimates the viscosity of the outer core matter. Interacting directly with the Earth's mantle the torques tend to stop the rotation. As the author supposes the Eckman time scale for the dissipation of transient fluid movements associated with unstable torques is much shorter than the time scale for regulating the rotation speed of the inner core and mantle in a stable regime of Couette flow. A stable tidal torque applied to the mantle contributes to the rotation of the inner core to be slightly faster relative to the mantle. The increase in the speed of rotation is proportional to the effective viscosity of the outer core and the value ofitstorque. The rate of super-rotation of the inner core relative to the mantle estimated by the author is about 1 degree per year, which corresponds to the effective viscosity of the outer core of $10^{3} \mathrm{~Pa} \cdot \mathrm{s}$. The possible influence of glacial cycles in tidal torques is shown [13] somewhat to complicate this pattern, but it does not change the order of viscosity values. The differential rate of rotation changes the viscosity value by about 2 times for each 100thousand-year glacial cycle.

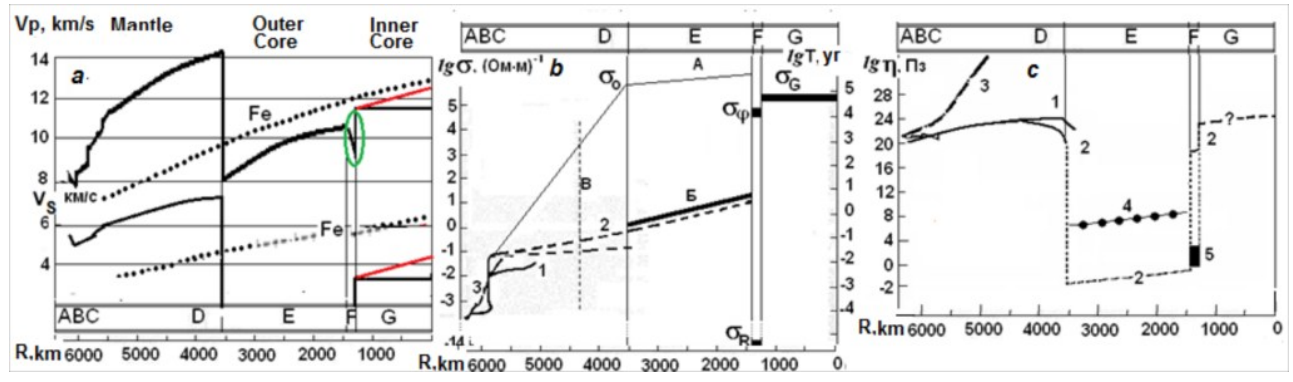

Fig. 5. (a) - Distribution of P-and S-waves velocity in the Earth core and mantle and in iron [1]. The common distribution of the waves velocity in the G-core (red). The distribution of the P-waves velocity for a phase transition (PT) in the F-layer (green). As measured the velocity of P-and S-waves in the inner core does not change (does not increase) approaching the center of the Earth. This fact is doubtless although as expected the waves velocity increases in the iron. The sound velocity would increase if the matter in the inner core is in its common state because both the density and temperature of the matter increase. (This phenomenon has no interpretation in the literature. Over the past 10 years, I have been following with increasing interest the papers in which quantum entangled (QE) matter is discussed. These papers and two my memoranda on quantum Geophysics are placed on my website www.vvkuz.ru. The matter of the Earth's inner core seems to me to be QE resulting in the unusual behavior of the sound velocity). QE matter is known to exhibit superconductivity and superfluidity which thereafter should be taken into account. Note that now only the matter of the inner core is QE although it was inherent to the Earth at the time of its formation. Apparently QE disappears when the matter of the inner core turns into the outer core through a phase transition. (b) The Earth's electrical conductance $(\sigma)$ in relation to the depth according to geomagnetic variations (1), secular variations (2) and magneto-telluric sounding (3) [9]. Interpolation of the curve 3 into the region of high $\sigma_{0}$, A- conductance in the outer iron core, jump $\left(\sigma_{0}\right)$ at the core - mantle boundary; B $\left(\sigma_{\circ}\right)$ of the outer core by our model; C-limit of oreliable values. Electrical conductance of the F-layer: $\left(\sigma_{\varphi}\right)$ - geostrophic flow, $\left(\sigma_{R}\right)$ - radial transport; $\left(\sigma_{\mathrm{G}}\right)$ - electrical conductance of the matter at the inner core. $T$ - time of the magnetic field diffusion (right scale). (c) - Viscosity distribution over the Earth: 1 - following to the Ranelli-Fischer model for adiabatic distribution of temperature in the mantle; 2 accepted distribution; 3 - distribution in the young Earth (before its differentiation); 4 - by seismology data [10]; 5 - viscosity of the F-layer (according to my model). 


\subsection{Superrotation in analysis of the splitting functions}

Despite very strict estimates of the relationship between the viscosity and the speed of differential rotation of the Earth's inner core, the differential rotation is assumed to be actually detected while studying the propagation features of bulk seismic waves. The inner core is revealed to rotate at a speed on $0.2^{\circ}-3^{\circ}$ per year faster than the Earth's crust and mantle. The wide variation in differences value of rotation rates is probably caused by the sensitivity of the studied waves to local (temporal and spatial) inhomogeneities in the structure of the inner core. On the other hand, as the natural oscillations, splitting functions, are insensitive to the local structure it is possible to estimate the value of the core differential rotation more accurately. Previous results on natural oscillationsshould be considered doubtful due to the relatively low quality of long-period digital data available 20 years ago. To obtain the most accurate data on the differential rotation of the core, the authors [12] analyzed natural oscillations as this method is insensitive to the source, i.e., the location and mechanism of the earthquake. As was concluded there is no differential rotation of the inner core with a rate of $\sim \pm 0.2 \%$ year. The inner core is supposed to be probably gravitationally "locked" by the Earth's mantle. There is some evidence to suggest [12] that essentially a superrotation of the core at a rate of $0.3 \%$ year is possible but a superrotation at a rate of $1 \%$ year contradicts all the data obtained.

By seismological data the superrotation of the inner core and the differential rotation of the outer core, one can conclude that the superrotation with relative rates of unit degrees per year is very doubtful. However, the time dependence of the anisotropy of the inner core may exist, and as it is related to the surface of the inner core, the presence of hydrodynamic transport is possible in the boundary region of the phase transition, i.e. in the F-layer.

The viscosity of the matter at the F-layer at a critical temperature is several orders of magnitude less than normal one. This is a well-known property of almost all substances. Superfluidity previously known does not bear on the real superfluidity of quantum liquids.

\subsection{PT in the F-layer}

As follow from the model of the hot Earth, in the region of the PT in the F-layer condensation and evaporation with its higher stage - boiling compete. The condensation rate $K\left(\mathrm{~s}^{-1} \mathrm{~cm}^{-3}\right)$ is given by the formula:

$$
K \approx 10^{26} \exp \left(-3 / \ln S_{\mathrm{v}}+\ln S_{\mathrm{v}}\right)
$$

here $S_{\mathrm{v}}$ is the supersaturation of vapour (the ratio of the vapor pressure in the system to that of saturated vapourat this temperature). The value of $S_{\mathrm{v}}$ is determined by the temperature of the vapor, its thermodynamic potential, the size of the droplet nuclei, the surface tension of liquid, etc. $K$ points to the number of elementary processes of particle fusion per an unit time in an unit volume. $K$ value determines the rate of droplets formation and their growth. In the evaporation (boiling) mode, vapor bubbles are mainly generated with the rate of formation:

$$
J\left(\mathrm{c}^{-1} \mathrm{~cm}^{-3}\right)=10^{37} \exp (-W / k T),
$$

where $W$ is the energy required to form a critical-size bubble. If at a temperature $T_{o}, K$ and $J$ become equal, then the system can, in principle, reach thermodynamic equilibrium. At the instant $t_{o}$ the system is overheated by $\Delta T$ to the temperature $T>T_{o}$ giving obviously the advantage to evaporation processes $(J>K)$, and a part of condensed matter would transform into the gaseous state. This process absorbs the heat of PT used for the formation, growth, and movement of bubbles yielding the system cooling. In the area of formation of "high-density" bubbles, negative pressure and empty space will arise, which immediately 
will be filled with the ambient matter and the Earth's volume will decrease. The heat will be removed from the PT zone by convection in the core and possibly in the mantle followed by the temperature $T$ decrease, and a condition in which $T<T_{o}$ may arise. At the point $T=$ $T_{o}$ a change of mode to the advantage of condensation $(K>J)$ will occur. Some matter will condense releasing the heat of PT and the system temperature increase. The cycle modes will repeat again.

\subsection{Separation of electric charges inside the F-layer}

Stabilized by PT the temperature of processes occurring in the F-layer is apparently close to the critical temperature of the substance. The substance of the inner core (let's assume that it is $\mathrm{SiO}_{2}$ ) an overpressured and superheated vapour (a supercritical liquid). Accompanied by a volume increase and the release of energy equal to the heat of PT the condensation of this substance leads to an increase in temperature $T_{E}$. At the border of the F-layer and the outer core $(\mathrm{E})$, silicon ions that carry a positive charge accumulate, and negative oxygen ions accumulate near the surface of the G-core. In the F-layer, a so-called double electric layer occurs. After $T_{E}$ becomes higher than $T_{G}$ the substance evaporates on the surface of the E-core. The evaporation occurs with the absorption of PT energy, while the polarity of the double layer reverses (Fig.6).

Consider the processes in the F-layer, bordered between the "solid" inner core and the "liquid" outer one. According to the Clausius-Clapeyron relation, the temperature distributes as a function of pressure:

$$
\frac{d T}{d p}=\frac{V_{V}-V_{L}}{U} T
$$

here $V_{V}$ and $V_{L}$ are specific volumes of vapour and liquid, $U$ is the heat of PT.

Usually $V_{V}>V_{L}, U>0$ and $d T / d p>0$, but if the overcompressed substance decompresses and condensates into a substance with normal density occur, then $V_{V}<V_{L}$, and $d T / d p<0$ (temperature reversal). As known if $T \approx T_{C R}, U \rightarrow 0$, large values of $d T / d p$ are possible.

The temperature of the $G$-core matter $T \approx(1-3) 10^{4} \mathrm{~K}$ suggests its partial ionization. The degree of ionization follows from the Saha formula for constant $k_{e}$ of ionization equilibrium:

$$
k_{e}=\frac{\alpha^{2}}{1-\alpha^{2}} p\left(\frac{2 \pi m}{h^{2}}\right)^{3 / 2}(k T)^{5 / 2} \exp \frac{e \varphi}{k T},
$$

where $p$ is the "gas" pressure equal to that at the center of the Earth $\left(3.5 \cdot 10^{6} \mathrm{~atm}\right), \alpha$ is the degree of ionization $(\alpha<<1), \varphi$ is the ionization potential $(\varphi \approx 12 \mathrm{eV})$. Then the degree of ionization is: $\alpha \leq 1 \%$ for the G-core $(\mathrm{k} T \approx 3 \mathrm{eV}), \alpha \leq 0.01 \%$ for the F-layer $(k T \approx 1 \mathrm{eV})$. Densities of electric charge: $n_{e} \approx 10^{20} \mathrm{~cm}^{-3}$ (at the G-core) and $10^{18} \mathrm{~cm}^{-3}$ (at the F-layer).

\section{EMF reversals}

\subsection{Reversal model}

Slightly differing from those published earlier [1-4] the model of the EMF generation and the model of its reversals proposed here suggest that the source of the Hall current is the surface of the G-core, which is plasma offering an unlimited source of charges both positive and negative. Another advantage of the new model is that as the substance on the surface of 
the G-core is in a state of superfluidity, and the long-lived Rossbyvortices which become the long-lived GMA capturing a part of the Hall current, are formed on such a surface.

Model of the EMF generation due to the daily rotation of the DEL, initiation of the weak field and its amplification by the Hall current are justified in the encyclopedia of Geomagnetism [5].

Our new model (Fig. 6) evidences, as proposed earlier, that FT-1 (condensationevaporation) occurs in the F-layer. If the rates of these processes are equal, the reversal of the EMF evolves. The left part of Fig. 6 shows the change in model parameters corresponding to the "condensation - expansion" mode, and the right part shows the "evaporation-compression" mode. The Earth during the modern Brunheschron of about 800 thousand years is at the"quiet" period of compression. It was Matuyamachron in the EMF before Brunhes when the Earth was expanding. This assumption that sooner or later there will come a period similar to the Matuyamachron (New Matuyama) will be justified further. Here we will justify the principal possibility for generating the EMF without invoking the Dynamo theory.

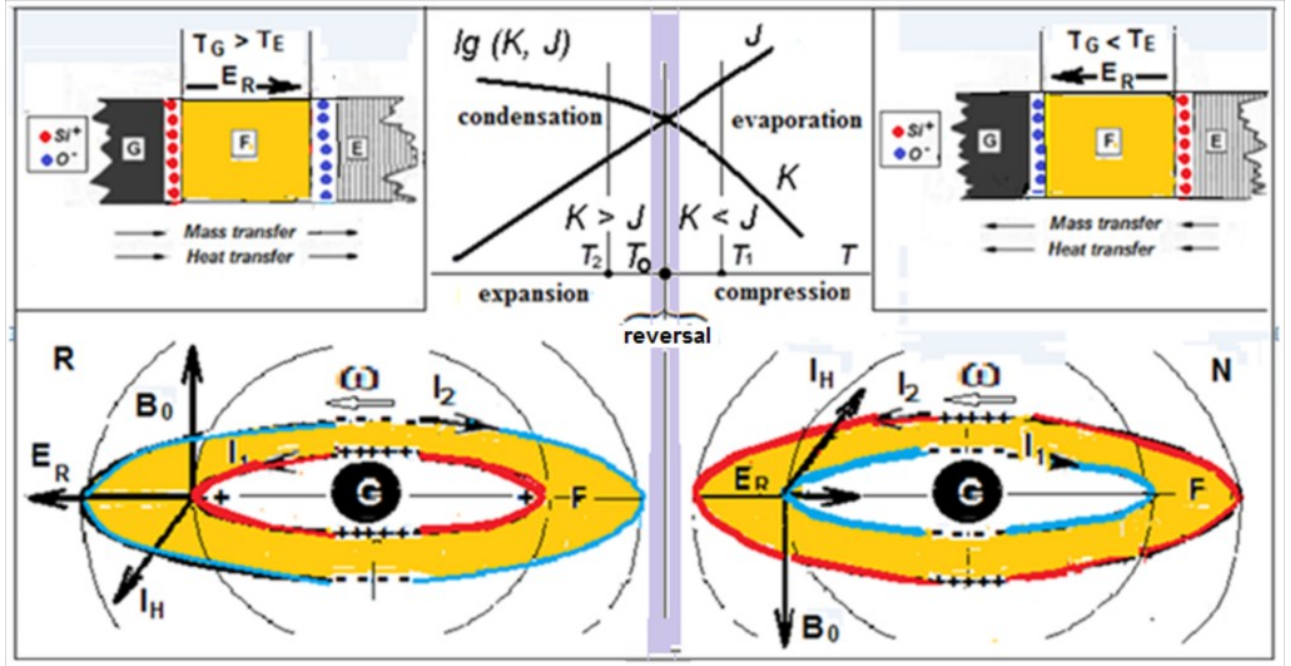

Fig. 6. Heat and mass transfer in the F-layer: expansion step $(K>J)$, compression step $(J>K) . K-$ the rate of condensation, $J$ - the rate of evaporation. Charges separation in the F-layer in the expansion step (left) and in the compression one (right). Formation of DEL, daily rotation and formation of the initial field $B_{0}$ at both cases. The interaction of the field $B_{0}$ with the radial electric field of the DEL $E_{R}$ causes the Hall current $I_{H}$, directed along the current which determines $B_{H}$.

\subsection{Nondynamo theories}

The encyclopedia of geomagnetism at the chapter Nondynamo theories ([5], P. 706) provides their four mechanisms: 1) Thermoelectric effect, 2) Thermomagnetic effect, 3) Hall effect and 4) Rotating electric field, and their four physical basis: 1) Dipole associated with a rotating ferromagnet, 2) Differences in temperature and composition, 3) Currents arising in presence of the magnetic field, 4) Current arising from the rotating charged capacitor.

The paper «Rotating electric fields and charges» ([5], P. 705, David J. Stevenson) placed here, states that EMF may be generated through the rotation of charges, the "problem is that one requires very large voltage differences in order to produce modest 
magnetic fields. In SI units, the electric field $E$ (in $\mathrm{V}^{\cdot} \mathrm{m}^{-1}$ ) required to explain a field of strength $\mathrm{B}$ (in tesla) is $\approx \mathrm{c}^{2} \mathrm{~B} / \mathrm{v}$, where $\mathrm{v}$ is the rotational velocity. This suggests $E \approx 10^{11} \mathrm{~V}$. $\mathrm{m}^{-1}$, which is physically impossible (it will ionize atoms) ». As one sees the generation of the EMF will require an electric field of a huge size. The note of D. J. Stevenson is quite true if the solution to the problem of the emergence of a magnetic field in the Earth's core is assumed only by the rotation of electric charges. In our case, the daily rotation of charges generates a very weak dipole field, which needs to be amplified by a current of Hall type directed along the current caused by the rotation of the DEL.

\subsection{Excursions of the EMF}

A geomagnetic excursion is an event lasting about ten thousand years, in which the virtual magnetic pole deviates from its position by about $60^{\circ}$, and then returns to its original position. If the polarity changes on about $180^{\circ}$, the intensity of the geomagnetic field significantly decreases. The difference between an excursion and a microchron is very conventional.
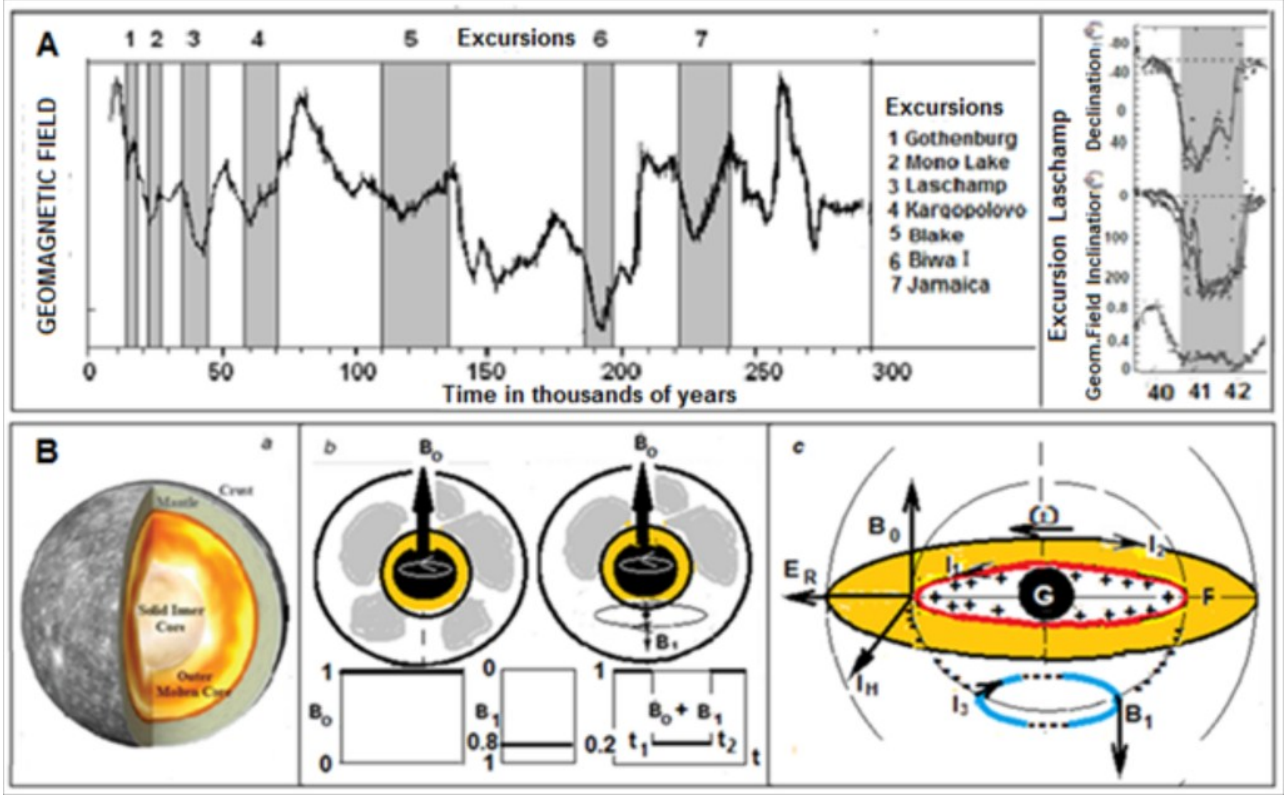

Fig 7. A: Excursions 1-7 (left), parameters of Laschamp excursion (right) [16].

B: Structure of the Mercury core (www.mercury-grail.com) - a. The inner core of the Earth - a black circle, the F-layer - the yellow ring, gray spots - ion plasma, constituting current rings -excursions. $B_{0}$ - the magnetic field of the Earth, $B_{1}$ - the magnetic field of the excursion. At the bottom - the addition of $B_{0}$ and $B_{1}-\mathrm{b}$. Core of the Earth, inner - G, F-layer (yellow). Direction of currents: $I_{l}$ and $I_{2}$ - the currents of DEL daily rotation, $I_{3}$ - the current creating the excursion, $I_{H}$-the Hall current, $B_{0}$ - the initial and main magnetic field of the Earth, $B_{l}$ - the magnetic field of the excursion, $E_{R}$ - the radial electric field of the DEL, $\omega$ - the direction of the Earth rotation - c.

The excursions in the Brunhesmagnetochron of more than 15 episodes are the most reliably identified. Excursions are the most mysterious phenomena in Geomagnetism. We have never encountered a plausible model of excursion in the literature on Geomagnetism, although we used this concept on our models of the excursions influence on climate and the 
life evolution $[14,15]$. It is still unknown what causes the EMF module to lower during an excursion. Let's see if we can find a plausible reason for an excursion onset in our EMF model.

Fig. 7-A shows some excursions of Brunheschron we discussed [14, 15]. Obtained as part of the NASA-GRAIL project a section of Mercury given at Fig. 7. B-a proves that that non-stationary flows of matter are observed in the outer liquid core of Mercury. This fact was used in our modeling an excursion. As assumed an accumulation of ion plasma which is formed from ions that are not used during the formation of the magnetic field of the excursion is possible in the space of the Earth's outer core.

Often during the Brunhes period with the modern polarity of EMF (N) the excursions are rare in epochs with the reverse (R) polarity. As noted above, the current that forms the EMF is the Hall current $\left(I_{N}\right)$, which flows along the surface of the inner core, and the charge carriers which are the ions, change depending on the direction of the $I_{N}$ : negative oxygen ions at the $\mathrm{N}$ polarity and positive silicon ions at the R polarity.

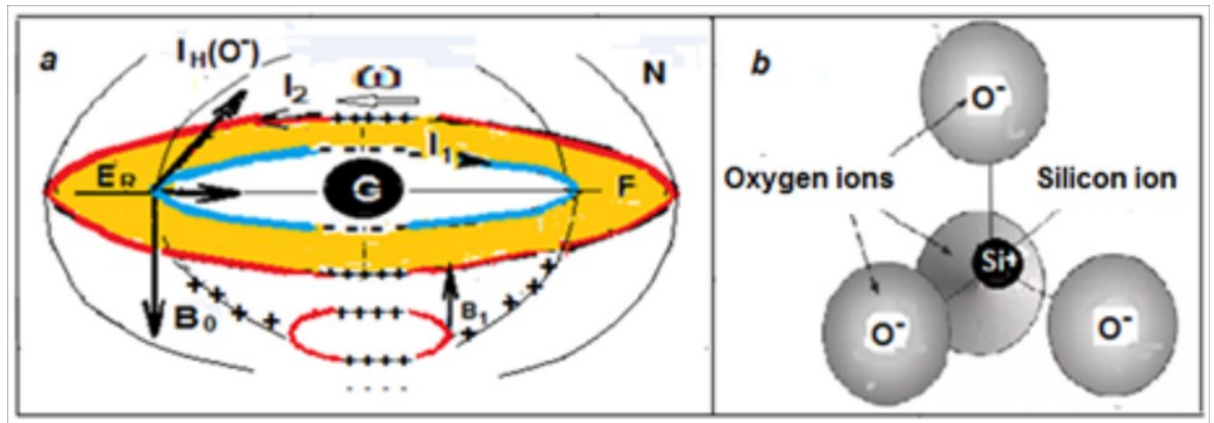

Fig. 8. Origin of an excursion during the Brunheschron $-a$, relative sizes of silicon and oxygen ions $-b$.

By this rule, remaining unused silicon ions make the ion plasma at the current polarity of EMF (N) (see Fig. 7B-b). Rotating together with the Earth free silicon ions drift along the boundary of the inner core, generating a current ring $\left(I_{3}\right)$ of the excursion and, respectively, the magnetic field with the sign reverse to that of the EMF, the value of which decreases, and the excursion is forming.

Why do excursions occur when silicon ions take part in their formation? Highly likely, it is of the mobility of silicon ions that is noticeably higher than that of oxygen ions. Figure 8-b shows that the size of oxygen ions significantly exceeds that of silicon ions. As the movement of an ion can be likened to that of a macroscopic ball in a viscous medium we apply the Stokes formula:

$$
u=\frac{z e}{6 \pi r \eta} \cdot \frac{E}{l},
$$

where $e$ is the electron charge; $z$ is the number of elementary charges of the ion; $r$ is the effective radius of the ion; $n$ is the viscosity coefficient; $E / l$ is the field intensity. This formula confirms that the mobility of ions is higher the smaller is their size.

The authors [17] were treating the geomagnetic field during some excursions of Brunheschron, including Lachamp and Mono Lake. EMF during these excursions decreased significantly and its polarity reversed. However, the field reversal did not occur, and after the excursion, the EMF polarity and its intensity then completely restored. The authors [17] wondered the result. It seemed to them that if the field changed its sign, the 
reversal should automatically start. The tackling to this problem follows from our model of EMF reversal which evidences that the reversal occurs (see Fig. 6) if $J=K$, when the rate of condensation is close to that of evaporation, and both $E_{R}$ and the dipole component of the EMF become zero. This point is the most important in our model of the excursion.

\section{Global magnetic anomalies and non-dipole part (NDP) of the EMF}

Global magnetic anomalies (GMA) are clearly seen on the map of the EMF full vector (F) (see Fig. 9-0). The intensity of the GMA field is so high that it can be compared with that of the dipole part of the EMF. Four anomalies are: Canadian (1), Brazilian (South Atlantic) (2), Siberian (3), and Southern (4) which combining it with the South magnetic pole is sometimes called the South pole, that is not quite true, and or the Antarctic. Gaussian expansion of the EMF tends to a maximum value of the first coefficient. Subtracting the first coefficients of the series from the general structure of the EMF, we get the so-called non-dipole part (NDP) in which map there are no well-defined anomalies. Apparently, this procedure is not correct if we want to understand what the EMF is. Moreover, GMA are well known to exist in space, the ionosphere and magnetosphere.

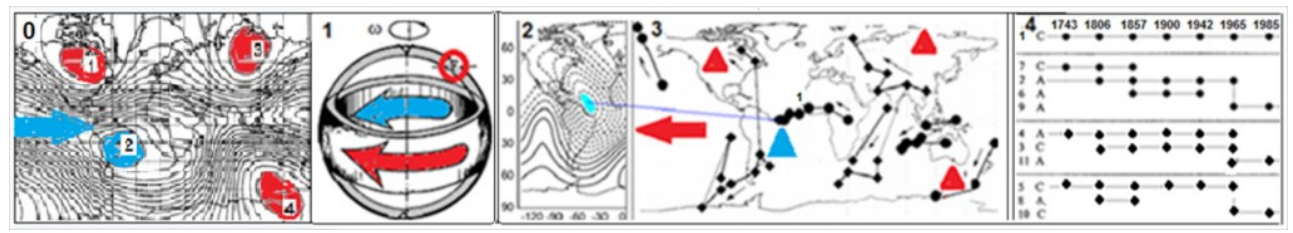

Fig. 9. GMA (0) and westward drift of NDP (2-4). 1 - superrotation of the G-core (blue arrow), drift of NDP (red arrow) on the surfaces of the F-layer. 2 - Brazilian NDP. $\mathbf{3}$ - the map of the NDP drift from 1743 to 1985 [19]. GMA (triangles). The Western transport (red arrow). 4 - the NDP numbers. Evolution of NDP: C-cyclones, A-anticyclones.

Table I

\begin{tabular}{|l|l|l|l|l|l|}
\hline Pipole & Central & Canadian & Siberian & Brazilian & Southern \\
\hline -oordinates & $78.3^{\circ} \mathrm{S} 106^{\circ} \mathrm{E}$ & $60^{\circ} \mathrm{N} 90^{\circ} \mathrm{W}$ & $60^{\circ} \mathrm{N} 120^{\circ} \mathrm{E}$ & $15^{\circ} \mathrm{S} 90^{\circ} \mathrm{W}$ & $70^{\circ} \mathrm{S} 150^{\circ} \mathrm{E}$ \\
\hline -alc. coord. & $63.3^{\circ} \mathrm{S} 25.3^{\circ} \mathrm{E}$ & $24^{\circ} \mathrm{N} 62.8^{\circ} \mathrm{W}$ & $45^{\circ} \mathrm{N} 66.8^{\circ} \mathrm{E}$ & $69.1^{\circ} \mathrm{S} 9.9^{\circ} \mathrm{W}$ & $72.5^{\circ} \mathrm{S} 133^{\circ} \mathrm{E}$ \\
\hline Md. $\times 10^{15} \mathrm{Tm}^{3}$ & 7.8 & 1.8 & 2.4 & 4.3 & 2,5 \\
\hline Pip. direct. & $\downarrow$ & $\downarrow$ & $\downarrow$ & $\uparrow$ & $\downarrow$ \\
\hline
\end{tabular}

EMF may be conceived as the sum of 5 dipoles: the Central and four GMA (Table I [11]). Here we consider GMA as Rossby vortices [18]. This approach revealed a striking similarity between GMA and long-lived vortices in the atmospheres of Jupiter and Saturn (Table II). For example, the Great Red Spot at Jupiter was discovered by Galileo more than 300 years ago. The mystery of vortices in the atmospheres of large planets like vorticesGMA in EMF has not yet been solved.

Table II

\begin{tabular}{|c|c|c|c|}
\hline Vortices of Jupiter & Vortices of Saturn & GMA & \\
\hline Large red spot $22^{\circ} \mathrm{S}$ & Big Bertha & Siberian $67^{\circ} \mathrm{N}$ & $\mathrm{A}$ \\
\hline Small red spot $19^{\circ} \mathrm{N}$ & Brown spot $42^{\circ} \mathrm{N}$ & Canadian $58^{\circ} \mathrm{N}$ & A \\
\hline Brownoval14 $4^{\circ} \mathrm{N}$ & UV spot & Brazilian $15^{\circ} \mathrm{S}$ & $\mathrm{C}$ \\
\hline White oval $34^{\circ} \mathrm{S}$ & Anna's Spot55 ${ }^{\circ} \mathrm{S}$ & South M. P55 ${ }^{\circ} \mathrm{S}$ & $\mathrm{A}$ \\
\hline
\end{tabular}


(The increasing the size of GMA nowadays may be attributed to the fact that despite the dipole part of the field tends to zero the total field continues to increase (Fig. 12).

\section{Drift of the North Magnetic Pole (NMP)}

Features of modern drift of NMP [22] are shown at Fig.10.
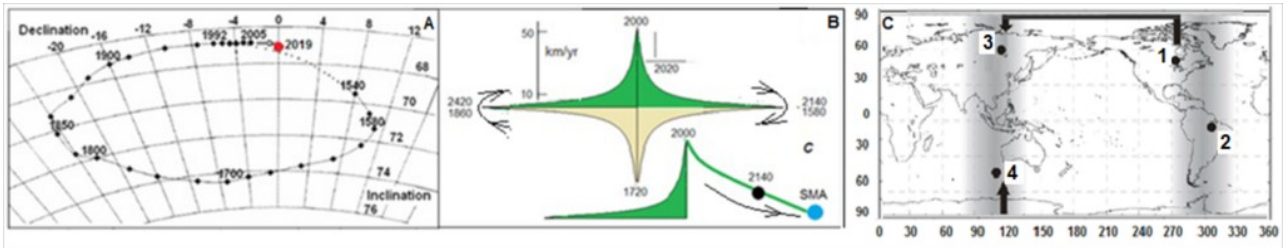

Fig. 10. A - declination $(D)$ and inclination $(I)$ measured at the Royal Observatory, Greenwich beginning in 1540 [20], B - velocity with the highest in 2000 and in (1720) 2280 of the NMP drifting over the cycle, the pole stopped in 1580 and 1860. It will stop in 2140 and 2420 . C - paths of magnetic polesdrift during reversals, 1-4 dark circles - GMA [1].

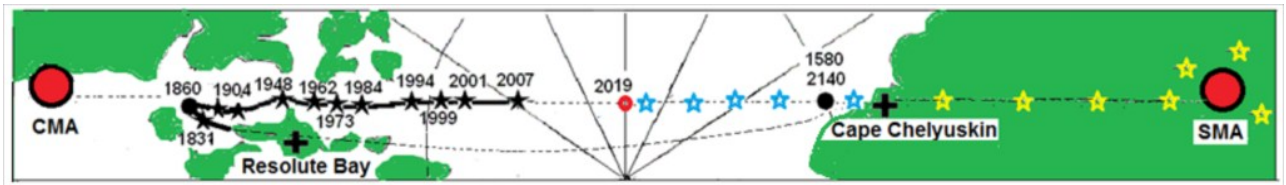

Fig. 11. NMP drift from 1831 up to 2019 and its possible drift in future.

The NMP should be located on the Siberian GMA at the beginning of the field reversal (3, Fig. 10-C). Nowadays the South magnetic pole (SMP), which drifted over Antarctica, is placed in the area of the GMA (4, Fig. 10-C). Note that the place and the time of its exit from Antarctica were predicted [21] by the Dumont d'Urville magnetic observatory built by France specially to fix the SMP on the coast.

As follows from Fig. 11, the NMP will cross the Arctic Ocean and in about 100 years (2140) it will stop near the Russian magnetic Observatory "Cape Chelyuskin". It would be nice (!) if the equipment of this Observatory which stopped its activity more than 20 years ago will be set up by the Russian magnetologists.

\section{How long the geomagnetic field will exist}

The model of the Hot expanding Earth allows us not only to explain plausibly the phenomena that occurred on Earth from the earliest time - the time of its formation, but also to predict what will happen to the Earth over the next 2-3 million years. It follows from the model that after the Brunhes period, during which the Earth was shrinking, a period of expansion similar to the Matuyama period (New Matuyama) will begin. Periods preceding the Matuyama period exceeded 1 million years, and Matuyama lasted almost 2 million years. The Brunheschron is about 800 thousand years long, therefore, it would continue for at least another 200 thousand years. It follows that the nearest reversal of the geomagnetic field actively discussed in connection with the increase in the rate of the NMP drift is unreal. 
My attention was drawn to Glatzmeier's figure [22] in which the upper part (Fig. 12) shows a decrease in the dipole component of the geomagnetic field, as well as an increase in its total field, quadrupole and octupole components. This picture corresponds to my interpretation of the modern geomagnetic field, when the dipole component decreases, and the total field and its components increase. It is the case that I have considered, when in addition to the dipole field, there is a field of anomalies, which increases due to the dipole field transforminginto the octupole one. To the Fig. 12 I added a reversal of the EMF, when $\mathrm{D}=0$, and the Hall currents are zero.

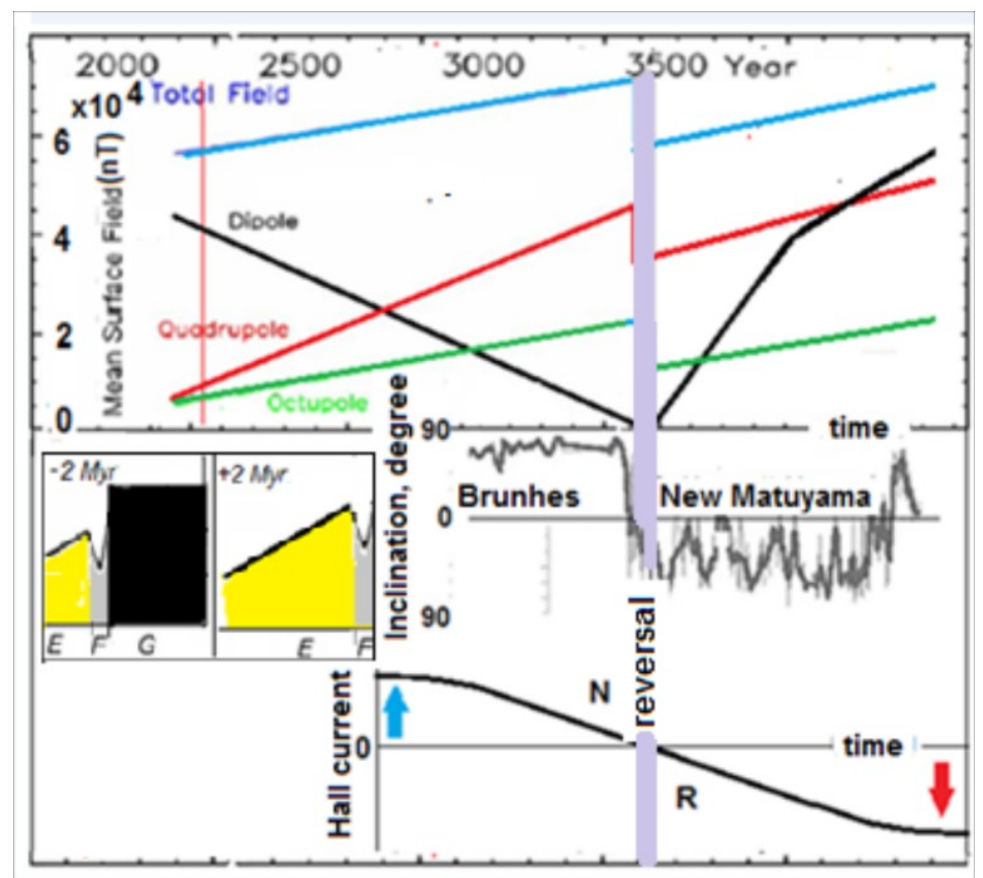

Fig. 12. Modeling of reversal after the Brunheschron in 3500 (according to Glassmeier et al., [22]). The reversal start after Glassmeier [22] (when the Dipole $=0$ ). Hall currents for $\mathrm{N}$-and R-polarities of the EMF, left bottom - the last Fig. in [2].

What will happen to the Earth after the end of its compression? As it follows from the model of the geomagnetic field of the Hot Expanding Earth a period of the Earth expansion will begin, and condensation of the matter in inner core will prevail over evaporation of that in the $\mathrm{G}$ - core. Turning into the liquid phase of the outer E-core the overcompressed matter of the inner core will be expanding during this decompression. The radius of the inner Gcore will decrease, and the resulting "excess" of matter will crystallize at the boundary between the core and the mantle, increasing its thickness and the radius of the Earth.

As from the model, the expansion of the Earth will stop after the matter of the inner core totally condenses. The inner core will disappear (the black rectangle in Fig. 12, -2 Myr), and the geomagnetic field generation will stop. The increase of the Earth radius $\Delta R$ will be about 20-30 km. It follows that the remaining time of the Earth's tectonic activity and its magnetic field, and, consequently, of the life on Earth, is no more than 2 million years.

As Glatzmeier et al. [22] obtained by extrapolating the decrease in the dipole component to the near future, the Earth's dipole field will disappear around 3500 (Fig.12) 
when the EMF reversal occurs, the Hall current changes its direction, and the magnetic pole starts its drift along the line connecting GMA 3 and 4 (Fig. 10-C) [18].

\section{Conclusions}

Starting my working on the Earth's physics about 40 years ago I cleared that A. Einstein considered the essence of the geomagnetic field and its reversed polarity one of the five main problems of physics. Discussing here Fig. 6-8, physics of the geomagnetic field, as well as the problem of changing its polarity become more understandable to me. This is just a model. Computer simulation of this phenomenon is necessary, and even better, direct observations of this amazing phenomenon, when the dipole $\mathrm{D}(\mathrm{N})=0$ will be replaced by $D(R)=0$ are necessary. As to Fig. 12, this can happen in about 1000 years and people (if there is no nuclear war or the destruction of humanity by another coronavirus) will observe this phenomenon. The first picture of the poles driftwas obtained 25 years ago [18], but a special understanding of the physics of the EMF was not achieved at that time. Recently I have become convinced that EMF can be generated without an iron Dynamo. This idea is clearly demonstrated in [5]. My new approach to the problem of reversals supported the essence of the excursions model, etc.

After the EMF model has become clearer, fundamentally new questions what should happen to the geomagnetic field when the Earth expands arise. These questions have never been raised by geophysicists in practical terms. It is obvious that it is necessary to understand physics of these important and fundamental changes in the Earth's evolution and prepare for them. Analysis of our model of the EMF generation suggests that sooner or later humanity will have to face the phenomena accompanying the expansion of the Earth and in addition to computer models, it is important to study past events that took place on Earth during the Matuyama chron.

The model of the EMF generation presented above alternative to the commonly accepted one. Working on various problems in the Earth physics for 40 years, I ensured there are huge, almost insurmountable difficulties in publishing a fundamentally new approach. Nevertheless, I consider it is necessary to introduce specialists-magnetologists into my results.

\section{References}

1. V.V. Kuznetsov, Introduction to the physics of the hot Earth (KamGU, PetropavlovskKamchatsky, 2008) (in Russian)

2. V.V. Kuznetsov, E3S Web of Conferences. 127, 02014(2019)

3. V.V. Kuznetsov, Problems of Geography. Modern Earth Geography 149, 93-143 (2019) (in Russian)

4. V.V.Kuznetsov, E3S Web of Conferences, 20, 02005 (2017)

5. Encyclopedia of Geomagnetism and Paleomagnetism. Ed. D. Gibbins and E. HerreroBervera. (Springer, Dordrecht, 2007).

6. C. Cavazzoni, G. L. Chiarotti, S. Scandoloet al., Science, 283, 44-46 (1999)

7. V.V. Kuznetsov, Phys. Usp. (1997) 40, 951 (in Russian)

8. V.V. Kuznetsov, Vestnik KRAUNC. Phys.-Mat. Science, 2(9), 36 (2014) (in Russian)

9. N.M. Rotanova, A.P. Pushkov, Deep electrical conductivity of the Earth (Moscow, Nauka, 1982) (in Russian)

10. G.G. Denisov,V.V. Novikov, Dokl. Ross. Nauk, 362, 484 (1998) (in Russian)

11. B.G. Bills, J. Geophys. Res, 104 , 2653-2666 (1999) 
12. G. Laske, G. Masters, Nature, 402, 66 (1999)

13. S. Lund, J.S. Stoner, J.E.T. Channel, G. Acton Physics of the Earth and Planetary Interiors, 156, 94-204 (2006)

14. N.D. Kuznetsova, V.V. Kuznetsov, http://kcs.dvo.ru/ikir/Russian/Science/2004/3-11.pdf (in Russian)

15. N.D. Kuznetsova, V.V. Kuznetsov, Vestnik TGU, 354, 199-204 (2012) (in Russian)

16. A. P. Roberts, Geoph. Res. Lett., 35, L17307 (2008)

17. M. Brown, M. Korteb, R. Holmec et al.,PNAS, 115, N. 20 5111-5116 (2018)

18. V.V. Kuznetsov, Dokl. Ross. Nauk., 340 (5). 685-687 (1995) (in Russian)

19. T. Yukutake, H.Tachinaka, Bull. Earthquake Res. Inst. 46, 1027-1074 (1968)

20. L.A. Bauer, Dissert. Univ. Berlin. (1895)

21. V.V. Kuznetsov, Dokl. Ross. Nauk. 361 (2), 248-251(1998) (in Russian)

22. K-H Glassmeier, A. Neuhaus, J.Vogt, Invited presentation Alpach Summer School Space Weather: Physics, Impacts and Predictions (2002). 